\title{
A GENERAL PRINCIPLE FOR LIMIT THEOREMS IN FINITELY ADDITIVE PROBABILITY
}

\author{
BY
}

RAJEEVA L. KARANDIKAR

\begin{abstract}
In this paper we formulate and prove a general principle which enables us to deduce limit theorems for sequences of independent random variables in a finitely additive setting from their analogues in the conventional countably additive setting.
\end{abstract}

1. Introduction. The first a.s. limit theorems in the finitely additive strategic setting were proved by Purves and Sudderth [10] and included a version of the strong law and the martingale convergence theorem. Chen $[4,5]$ proved some a.s. limit theorems for a sequence of independent random variables in the finitely additive strategic setting. Ramakrishnan [11] has proved the central limit theorem in the same set-up. In many of these proofs, the main technique was to 'approximate' the given sequence $\left\{Y_{k}\right\}$ of independent random variables by a sequence $\left\{Z_{k}\right\}$ such that each $Z_{k}$ takes only finitely many values. Behind these approximations, there is a general principle which we isolate and prove. Given the sequence $\left\{Y_{k}\right\}$ as above, we construct an independent sequence $\left\{X_{k}\right\}$ on a countably additive probability space which is 'very close' to $\left\{Y_{k}\right\}$ in distribution. To be more precise, we show that we can approximate $\left\{Y_{k}\right\}$ by $\left\{Z_{k}\right\}$ and $\left\{X_{k}\right\}$ by $\left\{W_{k}\right\}$ such that $\left\{Z_{k}\right\}$ and $\left\{W_{k}\right\}$ have the same distribution. Our main result is that 'a limit theorem' holds for $\left\{Y_{k}\right\}$ if and only if it holds for $\left\{X_{k}\right\}$. The formulation of this result is similar to Aldous' formulation of the 'subsequence principle' [1]. We show how to deduce the usual results like SLIN, WLLN, LIL, CLT and Donsker's and Strassen's invariance principles from our result. It should be pointed out that our method, although quite general, does not apply to prove results about martingales.

We now describe the finitely additive set-up with which we will be working.

Let $I$ be a nonempty set. Let $\left\{\gamma_{n}: n \geqslant 1\right\}$ be a sequence of finitely additive probability measures on all subsets of $I$. Let $H=I^{\infty}$ be equipped with the product of discrete topologies and let $A$ be the Borel $\sigma$ field of $H$. Using the methods of Dubins and Savage [6, 7] and Purves and Sudderth [10] it is possible to get a finitely additive probability $\sigma$ on $\underline{\underline{A}}$ (unique subject to certain regularity conditions) having the following desirable properties:

(i) $\sigma\left(A_{1} \times A_{2} \times \cdots\right)=\prod_{k=1}^{\infty} \gamma_{k}\left(A_{k}\right), A_{k} \subseteq I$.

Received by the editors May 7, 1981.

1980 Mathematics Subject Classification. Primary 60F05, 60G07.

Key words and phrases. Finitely additive probability, strategy, invariance principles.

(C)1982 American Mathematical Society $0002-9947 / 82 / 0000-0038 / \$ 03.50$ 
(ii) If $\underline{A}_{k}$ is a sequence of $\sigma$ fields on $I$ such that $\gamma_{k}^{\prime}=\gamma_{k}$ restricted to $\underline{A}_{k}$ is countably additive, then $\sigma=\gamma^{\prime}$ on $\underline{\underline{A}}{ }^{\prime}$ where $\underline{\underline{A^{\prime}}}=\otimes_{k} \stackrel{A}{\underline{A}}$ and $\gamma^{\prime}=\otimes_{k} \gamma_{k}^{\prime}$ (usual product measure).

(iii) Let $A_{n} \subset I$ and let $B_{n}=\left\{h \in H: h_{n} \in A_{n}\right\} \quad\left(h=\left(h_{1}, h_{2}, \ldots\right) \in H\right)$. Then $\sigma\left(\cap_{k=1}^{\infty} \cup_{n=k}^{\infty} B_{n}\right)=0$ if and only if $\sum_{n=1}^{\infty} \sigma\left(B_{n}\right)<\infty$. Further if $\sum_{n=1}^{\infty} \sigma\left(B_{n}\right)<\infty$, then $\sigma\left(\cup_{n=k}^{\infty} B_{n}\right) \rightarrow 0$ as $k \rightarrow \infty$.

(i) and (iii) follow from (ii). (ii) is a special case of Theorem 1, Purves and Sudderth [10].

2. Preliminaries and statement of the main result. Let $\mu$ be a finitely additive probability measure on $\left(R, \underline{B}_{R}\right)$ which is tight (i.e. $\left.\lim _{n} \mu([-n, n])=1\right)$. We will associate with $\mu$ a unique countably additive measure $v$ as follows:

$f \rightarrow \int f d \mu$ defines a positive linear functional on the space of bounded continuous functions from $R$ into itself. It follows easily from tightness of $\mu$ and Dini's theorem that this linear functional is $\sigma$-smooth (i.e. $f_{n} \downarrow 0$ implies $\int f_{n} d \mu \rightarrow 0$ ). Thus by Daniell's theorem [9, p. 60] there exists a unique countably additive probability measure $v$ on $\underline{\underline{B}}_{R}$ such that

$$
\int f d \mu=\int f d v
$$

for all bounded continuous functions $f$. The measure $v$ thus obtained is called the countably additive probability associated with $\mu$. The referee has pointed out that the countably additive measure $v$ is the "conventional companion" of Dubins and Savage [7, p. 190].

Let $f$ be a positive continuous function. Then for each $n$,

$$
\int(f \wedge n) d \mu=\int(f \wedge n) d v
$$

But

$$
\int f d \mu=\lim _{n} \int(f \wedge n) d \mu \quad \text { (definition) }
$$

and

$$
\int f d v=\lim _{n} \int(f \wedge n) d v \quad \text { (monotone convergence theorem). }
$$

Thus $\int f d \mu=\int f d v$.

From this it follows that, for a continuous function $f, \int f d \mu$ is well defined if and only if $\int f d v$ is and then $\int f d \mu=\int f d v$.

Let $F(x)=\mu((-\infty, x])$, and $G(x)=v((-\infty, x])$; we claim that $F(x)=G(x)$ if $x$ is a continuity point of $F$ or $G$. To see this, fix $x$ and for each $n \geqslant 1$ let $f_{n}, g_{n}$ be continuous functions such that

$$
1_{(-\infty, x-1 / n]} \leqslant f_{n} \leqslant 1_{(-\infty, x]} \leqslant g_{n} \leqslant 1_{(-\infty, x+1 / n]} .
$$

Then

$$
F\left(x-\frac{1}{n}\right) \leqslant \int f_{n} d \mu \leqslant F(x) \leqslant \int g_{n} d \mu \leqslant F\left(x+\frac{1}{n}\right)
$$


and

$$
G\left(x-\frac{1}{n}\right) \leqslant \int f_{n} d v \leqslant G(x) \leqslant \int g_{n} d v \leqslant G\left(x+\frac{1}{n}\right)
$$

Further, $\int f_{n} d \mu=\int f_{n} d v$ and $\int g_{n} d \mu=\int g_{n} d v$ for all $n$. Thus if $x$ is a continuity point of $F$ (or $G), F(x)=G(x)$.

Before we proceed to state our main result, we define convergence in distribution for 'random-elements' on a finitely additive probability space.

Let $H$ be a nonempty set, $\underline{\bar{A}}$ be a $\sigma$ field on $H$ and $\sigma$ be a finitely additive probability measure on $\underline{\underline{A}}$. Let $\overline{\bar{S}}$ be a metric space. Let $\xi_{k}: H \rightarrow S$ be a sequence of random elements in $S$ (i.e. $\xi_{k}^{-1} B \in \underset{=}{=}$ for all Borel sets in $S$ ) and let $\lambda$ be a countably additive probability measure on $S$.

DEFINITION. Say $\xi_{k}$ converge in distribution to $\lambda$ if $\sigma\left(\xi_{k} \in B\right) \rightarrow \lambda(B)$ for all Borel sets $B$ in $S$ such that $\lambda(\partial B)=0$.

The proof of the following theorem on equivalent conditions for convergence in distribution is the same as in the countably additive case (see [2, p. 11]) and hence is omitted.

THEOREM 1. Let $\left\{\xi_{k}\right\}$ be $S$ valued random elements on a finitely additive probability space $(H, A, \sigma)$ and let $\lambda$ be a countably additive probability measure on $S$.

Then the following are equivalent:

(i) $\int f\left(\xi_{k}\right) d \sigma \rightarrow \int f d \lambda$ for all bounded continuous functions $f$.

(ii) $\int f\left(\xi_{k}\right) d \sigma \rightarrow \int f d \lambda$ for all bounded uniformly continuous functions $f$.

(iii) $\lim \sup _{k} \sigma\left(\xi_{k} \in F\right) \leqslant \lambda(F)$ for all closed sets $F \subseteq S$.

(iv) $\lim \inf _{k} \sigma\left(\xi_{k} \in G\right) \geqslant \lambda(G)$ for all open sets $G \subseteq S$.

(v) $\xi_{k} \rightarrow \lambda$ in distribution.

The following proposition follows easily from the equivalence of (ii) and (v) in Theorem 1.

Proposition 2. Let $\left\{\xi_{k}\right\},\left\{\eta_{k}\right\}$ be random elements in $S($ on $(H, \underline{=}, \sigma))$ and let $d\left(\xi_{k}, \eta_{k}\right) \rightarrow 0$ in $\sigma$-probability. Then $\xi_{k}$ converges in distribution to $\lambda$ implies that $\eta_{k}$ converges in distribution to $\lambda$.

Let $I,\left\{\gamma_{n}\right\}, H, A, \sigma$ be as in the introduction. Let $\left\{f_{k}: k \geqslant 1\right\}$ be a sequence of mappings from $I$ into $R$. Let $Y_{k}(h)=f_{k}\left(h_{k}\right)$ be the coordinate map on $H$ induced by $f_{k}$ (here $\left.h=\left(h_{1}, h_{2}, \ldots\right) \in H\right)$. Let $\mu_{k}$ be the distribution of $Y_{k}$, i.e. $\mu_{k}(A)=$ $\sigma\left(Y_{k} \in A\right), A \in \underline{\underline{B}}_{R}$. Assume that $\mu_{k}$ is tight for each $k$ (i.e. $\left.\sigma\left(\left|Y_{k}\right| \geqslant n\right) \rightarrow 0 \forall k\right)$. Let $v_{k}$ be the countably additive measure associated with $\mu_{k}$. Let $(\Omega, \underline{F}, P)$ be a (countably additive) probability space supporting a sequence $\left\{X_{k}\right\}$ of independent random variables such that $P X_{k}^{-1}=v_{k}$ for all $k$.

Let $Y=\left(Y_{1}, Y_{2}, \ldots\right)$ and $X=\left(X_{1}, X_{2}, \ldots\right)$ be the random vectors taking values in $R^{\infty}$ corresponding to the sequence $\left\{Y_{k}\right\}$ and $\left\{X_{k}\right\}$ respectively. 
THEOREM 3. Fix $0<p<\infty$.

(a) Let $A \in \underline{\underline{B}}^{\infty}\left(\right.$ Borel $\sigma$ field of $\left.R^{\infty}\right)$ be such that

$$
x \in A \text { and } \sum_{i=1}^{\infty}\left|x_{i}-x_{i}^{\prime}\right|^{p}<\infty \quad \text { implies } x^{\prime} \in A .
$$

Then $Y \in A$ a.s. $\sigma$ if and only if $X \in A$ a.s. $P$.

(b) Let $(S, d)$ be a separable metric space and $\left\{g_{k}: k \geqslant 1\right\}$ be a sequence of measurable mappings from $R^{\infty}$ into $S$ such that

$$
d\left(g_{k}(x), g_{k}\left(x^{\prime}\right)\right) \leqslant \sum_{i=1}^{\infty} C_{k, i}\left|x_{i}-x_{i}^{\prime}\right|^{p}
$$

where $C_{k, i}$ are positive constants bounded by 1 such that for each $i, \lim _{k} C_{k, i}=0$.

Let $s \in S$. Then $g_{k}(Y) \rightarrow s$ in $\sigma$-probability if and only if $g_{k}(X) \rightarrow s$ in P-probability.

(c) Let $\lambda$ be a countably additive probability measure on $S$ and $\left\{g_{k}\right\}$ be as in (b) satisfying (2). Then $g_{k}(Y) \rightarrow \lambda$ in distribution if and only if $g_{k}(X) \rightarrow \lambda$ in distribution.

REMARK 1. If $\left\{g_{k}\right\}$ satisfy (2), then $A=\left\{x: g_{k}(x) \rightarrow s\right\}$ satisfies (1) and hence (a) implies that $g_{k}(Y) \rightarrow s$ a.s. $\sigma$ if and only if $g_{k}(X) \rightarrow s$ a.s. $P$.

3. Proofs. The idea of the proof is to approximate $Y=\left(Y_{1}, Y_{2}, \ldots\right)$ by $Z=$ $\left(Z_{1}, Z_{2}, \ldots\right)$ and $X=\left(X_{1}, X_{2}, \ldots\right)$ by $W=\left(W_{1}, W_{2}, \ldots\right)$ such that the distribution of $Z$ under $\sigma$ is the same as the distribution of $W$ under $P$.

Let $F_{k}(x)=\mu_{k}((-\infty, x]), G_{k}(x)=v_{k}((-\infty, x])$ and $D_{k}=\{x: x$ is a continuity point of $F_{k}$. Then as observed earlier $F_{k}(x)=G_{k}(x)$ for all $x \in D_{k}$.

For each $k \geqslant 1$, choose numbers $\left\{a_{k, j}: 0 \leqslant j \leqslant n_{k}\right\}$ belonging to $D_{k}$ such that

(i) $F_{k}\left(a_{k, n_{k}}\right)-F_{k}\left(a_{k, 0}\right) \geqslant 1-1 / 2^{k}$ and

(ii) $0<a_{k, j}-a_{k, j-1}<1 / 2^{k}, 1 \leqslant j \leqslant n_{k}$.

This can be done as for each $k, \lim _{x \rightarrow \infty} F_{k}(x)=1, \lim _{x \rightarrow-\infty} F_{k}(x)=0$ and $D_{k}$ complement is at most countable.

Define $Z=\left(Z_{1}, Z_{2}, \ldots\right)$ and $W=\left(W_{1}, W_{2}, \ldots\right)$ by

$$
\begin{aligned}
Z_{k}(h) & =a_{k, 0} & & \text { if } Y_{k}(h) \leqslant a_{k, 0}, \\
& =a_{k, j} & & \text { if } a_{k, j}<Y_{k}(h) \leqslant a_{k, j+1}: 0 \leqslant j<n_{k}, \\
& =a_{k, n_{k}} & & \text { if } a_{k, n_{k}}<Y_{k}(h),
\end{aligned}
$$

and

$$
\begin{aligned}
W_{k}(w) & =a_{k, 0} & & \text { if } X_{k}(w) \leqslant a_{k, 0}, \\
& =a_{k, j} & & \text { if } a_{k, j}<X_{k}(w) \leqslant a_{k, j+1}: 0 \leqslant j<n_{k}, \\
& =a_{k, n_{k}} & & \text { if } a_{k, n_{k}}<X_{k}(w) .
\end{aligned}
$$

LEMMA 1. $\sigma(Z \in B)=P(W \in B)$ for all Borel sets $B$ in $R^{\infty}$.

Proof. First observe that, for each $k$,

$$
\begin{aligned}
\sigma\left(Z_{k}=a_{k, j}\right) & =F_{k}\left(a_{k, j+1}\right)-F_{k}\left(a_{k, j}\right)=G_{k}\left(a_{k, j+1}\right)-G_{k}\left(a_{k, j}\right) \\
& =P\left(W_{k}=a_{k, j}\right), \quad 1 \leqslant j<n_{k} .
\end{aligned}
$$

Similarly $\sigma\left(Z_{k}=a_{k, 0}\right)=P\left(W_{k}=a_{k, 0}\right)$ and $\sigma\left(Z_{k}=a_{k, n_{k}}\right)=P\left(W_{k}=a_{k, n_{k}}\right)$. 
For each $k$, let $\underline{\underline{A}}_{k}$ be the finite field on $I$ generated by the sets $\left\{f_{k} \leqslant a_{k, 0}\right\}$, $\left\{f_{k}>a_{k, n_{k}}\right\},\left\{a_{k, j}<f_{k} \leqslant a_{k, j+1}\right\}$. Let $\gamma_{k}^{\prime}$ be $\gamma_{k}$ restricted to $\underline{A}_{k}$. Then (trivially) $\gamma_{k}^{\prime}$ is countably additive. Let $\underline{\underline{A}}^{\prime}=\otimes \underline{\underline{A}}_{k}$ and $\gamma=\otimes \gamma_{k}^{\prime}$ (the usual product measure on $\left.\left(H, A^{\prime}\right)\right)$. Then by usual arguments, it follows that $\gamma^{\prime}(Z \in B)=P(W \in B)$ for all Borel sets $B$ in $R^{\infty}$. Since $\sigma$ agrees with $\gamma^{\prime}$ on $\underline{\underline{A}}^{\prime}$ (property (ii) of $\sigma$ ) the proof is complete.

The next lemma shows that $Z(W)$ approximates $Y(X)$ 'nicely'.

LeMMA 2. For $0<p<\infty$

(a) $\sum_{k=1}^{\infty}\left|Y_{k}-Z_{k}\right|^{p}<\infty$ a.s. o.

(b) $\sum_{k=1}^{\infty}\left|X_{k}-W_{k}\right|^{p}<\infty$ a.s. $P$.

(c) $d\left(g_{k}(Y), g_{k}(Z)\right) \rightarrow 0$ in $\sigma-p r o b a b i l i t y$.

(d) $d\left(g_{k}(X), g_{k}(W)\right) \rightarrow 0$ in P-probability.

Proof. (a) $\sigma\left(\left|Y_{k}-Z_{k}\right| \geqslant 1 / 2^{k}\right) \leqslant F_{k}\left(a_{k, n_{k}}\right)-F_{k}\left(a_{k, 0}\right) \leqslant 1 / 2^{k}$; hence by the Borel-Cantelli lemma (property (iii) of $\sigma$ )

$$
\sigma\left(\left|Y_{k}-Z_{k}\right| \geqslant \frac{1}{2^{k}} \text { i.o. }\right)=0 .
$$

Now (a) follows from this.

(b) follows similarly as $P\left(\left|X_{k}-W_{k}\right| \geqslant 1 / 2^{k}\right) \leqslant 1 / 2^{k}$.

(c) By the Borel-Cantelli lemma (property (iii) of $\sigma$ ),

$$
\sigma\left(\left|Y_{k}-Z_{k}\right| \geqslant 1 / 2^{k} \text { for some } k \geqslant n\right) \rightarrow 0 \text { as } n \rightarrow \infty .
$$

We now show that this and (2) imply (c). Given $\varepsilon>0$ and $\delta>0$ choose $N$ such that

$$
\frac{1}{2^{N p}\left(1-2^{-p}\right)}<\frac{\varepsilon}{2} \text { and } \sigma\left(\left|Y_{k}-Z_{k}\right|>\frac{1}{2^{k}} \text { for some } k \geqslant N\right)<\frac{\delta}{2} \text {. }
$$

For each $i$, since the distribution of $Y_{i}-Z_{i}$ is tight and $\lim _{k} C_{k, i}=0$ for each $i$,

$$
\sigma\left(\sum_{i=1}^{N} C_{k, i}\left|Y_{i}-Z_{i}\right|^{p} \geqslant \frac{\varepsilon}{2}\right) \rightarrow 0 \text { as } k \rightarrow \infty .
$$

Choose $k_{0}$ large enough such that

$$
\sigma\left(\sum_{i=1}^{N} C_{k, i}\left|Y_{i}-Z_{i}\right|^{p} \geqslant \frac{\varepsilon}{2}\right) \leqslant \frac{\delta}{2} \quad \text { if } k \geqslant k_{0} .
$$

Then for $k \geqslant k_{0}$,

$$
\begin{aligned}
\sigma\left(d\left(g_{k}(Z), g_{k}(Y)\right) \geqslant \varepsilon\right) & \leqslant \sigma\left(\sum_{i=1}^{\infty} C_{k, i}\left|Y_{i}-Z_{i}\right|^{p} \geqslant \varepsilon\right) \\
& \leqslant \sigma\left(\sum_{i=1}^{N} C_{k, i}\left|Y_{i}-Z_{i}\right|^{p} \geqslant \frac{\varepsilon}{2}\right)+\frac{\delta}{2} \leqslant \delta .
\end{aligned}
$$

Hence $d\left(g_{k}(Z), g_{k}(Y)\right) \rightarrow 0$ in $\sigma$-probability.

(d) follows similarly.

The theorem now follows easily from these two lemmas. 
PROOF OF THE THEOREM.

(a)

$$
\begin{aligned}
Y \in A \text { a.s. } \sigma & \Leftrightarrow Z \in A \text { a.s. } \sigma \quad \text { by (1) and Lemma 2(a) } \\
& \Leftrightarrow W \in A \text { a.s. } P \quad \text { by Lemma } 1 \\
& \Leftrightarrow X \in A \text { a.s. } P \quad \text { by (1) and Lemma 2(b). }
\end{aligned}
$$

(b) $g_{k}(Y) \rightarrow s$ in $\sigma$ probability $\Leftrightarrow g_{k}(Z) \rightarrow s$ in $\sigma$-probability by Lemma $2($ c)

$$
\Leftrightarrow g_{k}(W) \rightarrow s \text { in } P \text {-probability by Lemma } 1
$$$$
\Leftrightarrow g_{k}(X) \rightarrow s \text { in } P \text {-probability by Lemma } 2(\mathrm{~d}) \text {. }
$$

(c) $g_{k}(Y) \rightarrow \lambda$ in distribution $\Leftrightarrow g_{k}(Z) \rightarrow \lambda$ in distribution by Lemma 2(c)

$$
\begin{aligned}
& \Leftrightarrow g_{k}(W) \rightarrow \lambda \text { in distribution by Lemma } 1 \\
& \Leftrightarrow g_{k}(X) \rightarrow \lambda \text { in distribution by Lemma } 2(\mathrm{~d}) .
\end{aligned}
$$

4. Consequences. We have already observed that $\int f\left(Y_{k}\right) d \sigma=\int f\left(X_{k}\right) d P$ (if either of the two integrals is well defined) for all continuous functions $f$. Thus limit theorems for the sequence $\left\{X_{k}\right\}$ whose hypotheses involve only the moments of $\left\{X_{k}\right\}$ follow for the sequence $\left\{Y_{k}\right\}$ by virtue of our theorem. (With the additional assumption that each ' $Y_{k}$ ' is tight. In most of the situations, the other conditions will imply tightness of ' $Y_{k}$ '.) This includes Kolmogorov's strong law, Khinchin's weak law (in the finitely additive setting, strong law does not imply weak law!) and the Hartman-Wintner law of iterated logarithm. We illustrate by an example.

TheOREM (CHEN) SLLN. Let $\left\{Y_{k}\right\}$ be identically distributed $\left(\mu_{k}=\mu_{1}\right.$ for all $\left.k\right)$, $\int\left|Y_{1}\right| d \sigma<\infty$ and $\int Y_{1} d \sigma=0$. Then

$$
\frac{Y_{1}+\cdots+Y_{n}}{n} \rightarrow 0 \text { a.s. } \sigma .
$$

Proof. Take $A=\left\{x \in R^{\infty}:\left(x_{1}+\cdots+x_{n}\right) / n \rightarrow 0\right\}$.

Check that $A$ satisfies (1) of Theorem 3. Then, $Y \in A$ a.s. $\sigma$ if and only if $X \in A$ a.s. $P$. Further $\mu_{k}=\mu_{1} \forall k$ implies $X_{k}$ are identically distributed,

$$
\int\left|X_{1}\right| d P=\int\left|Y_{1}\right| d P<\infty, \quad \int X_{1} d P=\int Y_{1} d \sigma=0 .
$$

Hence (by Kolmogorov's strong law-countably additive version [3, p. 52]) $X \in A$ a.s. $P$. This implies that $Y \in A$ a.s. $\sigma$.

In many other situations, say the Lindeberg-Feller central limit theorem or Kolmogorov's three series theorem, the conditions on $X_{k}$ can be transformed into equivalent conditions involving $\int f\left(X_{k}\right) d P$ for some continuous functions $f$ and hence again the finitely additive versions can be derived from their countably additive analogues. We illustrate this by an example.

THEOREM (RAMAKRISHNAN) (CLT). Let $\int Y_{k} d \sigma=0$, and let $v_{k}^{2}=\int Y_{k}^{2} d \sigma, s_{n}^{2}=v_{1}^{2}$ $+\cdots+v_{k}^{2}$. Then

(i) $\left(Y_{1}+\cdots+Y_{n}\right) / s_{n} \rightarrow N(0,1)$ in distribution and

(ii) $\max _{k \leqslant n}\left(v_{k}^{2} / s_{n}^{2}\right) \rightarrow 0$ 
if and only if the Lindeberg condition (L) holds for $Y_{n}$ :

$$
\frac{1}{s_{n}^{2}} \sum_{k=1}^{n} \int_{\left\{\left|Y_{k}\right| \geqslant \varepsilon s_{n}\right\}}\left|Y_{k}\right|^{2} d \sigma \rightarrow 0 \text { as } n \rightarrow \infty \text { for all } \varepsilon>0 \text {. }
$$

Proof. Let $g_{k}(x)=\left(x_{1}+\cdots+x_{k}\right) / s_{k}$ for $x \in R^{\infty}$. Then

$$
\left|g_{k}(x)-g_{k}\left(x^{\prime}\right)\right| \leqslant \sum_{i=1}^{k} \frac{1}{s_{k}}\left|x_{i}-x_{i}^{\prime}\right| \text {. }
$$

Thus if $s_{n}^{2} \rightarrow \infty$, then (2) holds with $C_{k, i}=\left(1 / s_{k}\right)$. Thus, if $s_{n}^{2} \rightarrow \infty$, then by Theorem $3, g_{k}(Y) \rightarrow N(0,1)$ in distribution if and only if $g_{k}(X) \rightarrow N(0,1)$ in distribution.

For each $n \geqslant 1, \varepsilon>0$, fix a continuous function $f_{n, \varepsilon}$ such that

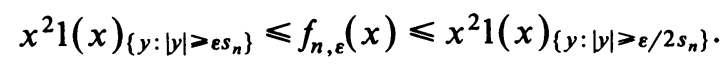

Then

$$
\int_{\left\{\left|Y_{k}\right| \geqslant \varepsilon s_{n}\right\}}\left|Y_{k}\right|^{2} d \sigma \leqslant f_{n, \varepsilon}\left(Y_{k}\right) d \sigma \leqslant \int_{\left\{\left|Y_{k}\right| \geqslant \varepsilon / 2 s_{n}\right\}}\left|Y_{k}\right|^{2} d \sigma
$$

and hence $(\mathrm{L})$ holds for $Y$ (respectively $X$ ) if and only if $\left(\mathrm{L}^{\prime}\right)$ holds for $Y$ (respectively $X$ ), where $\left(\mathrm{L}^{\prime}\right)$ is

$$
\frac{1}{s_{n}^{2}} \sum_{k=1}^{n} \int f_{n, \varepsilon}\left(Y_{k}\right) d \sigma \rightarrow 0 \text { for all } \varepsilon>0 .
$$

But $\left(\mathrm{L}^{\prime}\right)$ holds for $Y$ if and only if $\left(\mathrm{L}^{\prime}\right)$ holds for $X$. Thus $(\mathrm{L})$ holds for $Y$ if and only if (L) holds for $X$.

Now, the result follows from the Lindeberg-Feller theorem-countably additive case $[8$, p. 280].

We now show that Donsker's invariance principle and Strassen's invariance principle can be proved in the finitely additive setting.

THEOREM (DONSKER'S INVARIANCE PRINCIPLE). For each $n$, define $g_{n}: R^{\infty} \rightarrow C[0,1]$ as follows:

$$
g_{n}(x)(t)=\frac{x_{1}+\cdots+x_{k}}{\sqrt{n}} \quad \text { if } t=\frac{k}{n} \text {, linear elsewhere. }
$$

Let $Y_{1}, Y_{2}, \ldots$ have identical distributions, $\int Y_{1} d \sigma=0, \int Y_{1}^{2} d \sigma=1$. Then $g_{n}(Y)$ converges in distribution to the Wiener measure.

Proof. Observe that

$$
d\left(g_{n}(x), g_{n}\left(x^{\prime}\right)\right)=\sup _{t}\left|g_{n}(x)(t)-g_{n}\left(x^{\prime}\right)(t)\right| \leqslant \sum_{k=1}^{n} \frac{1}{\sqrt{n}}\left|x_{k}-x_{k}^{\prime}\right| \text {. }
$$

Thus $g_{k}$ satisfies (2).

Now, by Donsker's theorem (countably additive version [2, p. 68]), $g_{k}(X)$ converges in distribution to Wiener measure and hence by Theorem 3 , so does $g_{k}(Y)$. 
THEOREM (STRASSEN'S INVARIANCE PRINCIPLE). Let $Y_{1}, Y_{2}, \ldots$ have identical distributions, $\int Y_{1} d \sigma=0, \int Y_{1}^{2} d \sigma=1$. Then a.s. $\sigma$, the sequence of functions $g_{n}(Y) / \sqrt{\log \log n}$ is relatively compact in $C[0,1]$ and its set of limit points is $K=$ $\left\{f \in C[0,1]: f\right.$ absolutely continuous, $f(0)=0$ and $\left.\int_{0}^{1} f^{\prime}(u)^{2} d u \leqslant 1\right\}$ (here $g_{n}$ are as in the previous theorem).

Proof. Let $A=\left\{x: g_{n}(x) / \sqrt{\log \log n}\right.$ is relatively compact in $C[0,1]$ and set of its limit point is $K$.

Then, it can be seen that $A$ satisfies (1) and thus the result follows from Strassen's invariance principle (countably additive version [12]) and Theorem 3 .

REMARK. Let $F_{k}$ be a sequence of nondecreasing functions on $R$ such that $\lim _{x \rightarrow-\infty} F_{n}(x)=0$ and $\lim _{x \rightarrow \infty} F_{n}(x)=1$. Let $\underline{\underline{C}}$ be the field of subsets of $R$ generated by the family $\{(a, b]:-\infty \leqslant a<b \leqslant \infty\}$. Each $F_{k}$ defines a unique finitely additive 'tight' probability measure on the field $\underline{\underline{C}}$ by the formula $\mu_{k}((-\infty, b])=F_{k}(b)$.

Consider the field $\underline{\underline{D}}$ of subsets of $R^{\infty}$ defined by

$$
\underline{\underline{D}}=\bigcup \bigotimes_{k} \underline{\underline{A_{k}}} k
$$

where the union is taken over all sequences $\left\{\underline{\underline{A}}_{k}\right\}$ of finite subfields of $\underline{\underline{C} \text {. For such a }}$ sequence $\left\{\underline{\underline{A}}_{k}\right\}$, each $\mu_{k}$ restricted to $\underline{\underline{A}} k$ is (trivially) countably additive. Let $\lambda_{0}$ be the usual countably additive product measure on $\otimes_{k} \underline{A}_{k}$ with marginals $\mu_{k}$ (restricted to $\underline{\underline{A}}_{k}$ ). It can be easily seen that these measures are consistent and hence determine a unique finitely additive measure $\lambda$ on $\underline{\underline{D}}$. Let $\sigma$ be any extension of $\lambda$ to $\underline{\underline{B}}^{\infty}$. Then (by construction of $\lambda$ ) it follows that

(ii)' For any sequence $\left\{\underline{\underline{A}}_{k}\right\}$ of finite subfields of $\underline{\underline{C}}, \sigma$ restricted to $\otimes_{k} \underline{\underline{A}}_{k}$ agrees with $\otimes_{k} \mu_{k}^{\prime}$ where $\mu_{k}^{\prime}$ is $\mu_{k}$ restricted to $\underline{A}_{k}$.

Now let $Y_{k}$ be the coordinate random variables on $\left(R^{\infty}, \underline{\underline{B}}^{\infty}, \sigma\right)$.

Let $G_{k}(x)=\lim _{y \downarrow x} F_{k}(y)$ for $x \in R, k \geqslant 1$, and let $(\overline{\bar{\Omega}}, \underline{\underline{F}}, P)$ be a probability space supporting a sequence $\left\{X_{k}\right\}$ of independent random variables with $P\left(X_{k} \leqslant x\right)$ $=G_{k}(x)$ for all $x \in R, k \geqslant 1$.

Then it follows easily (by using the property (ii)' of $\sigma$ instead of (ii)) that Theorem 3 (and hence its consequences) is true in this setting as well.

5. Let $(S, d)$ be a separable metric space and $\mu$ be a finitely additive tight probability measure on $\underline{B}_{S} . f \rightarrow \int f d \mu$ defines a $\sigma$-smooth positive linear functional on the space of bounded continuous functions from $S$ into $R$ and hence by Daniell's theorem [9, p. 60] there exists a unique countably additive probability measure $v$ on $\underline{B}_{S}$ such that $\int f d \mu=\int f d v$ for all $f \in C_{b}(S)$. Theorem 1 implies that $\mu(A)=v(A)$ if $v(\partial A)=0$.

Let $(H, \underline{\underline{A}}, \sigma)$ be as in the introduction. Let $f_{k}: I \rightarrow S$, and $Y_{k}(h)=f_{k}\left(h_{k}\right)$. Let $\mu_{k}(B)=\sigma\left(\bar{Y}_{k} \in B\right)$. Assume $\mu_{k}$ is tight for each $k$. Let $v_{k}$ be the countably additive measure associated with $\mu_{k}$ as above. Let $\left\{X_{k}\right\}$ be an independent sequence of $S$ valued random elements on a countably additive probability space $(\Omega, \underline{\underline{F}}, P)$ such that $v_{k}(B)=P\left(X_{k} \in B\right)$.

The following theorem is an analogue of Theorem 3. 
THEOREM. Fix $0<p<\infty$.

(a) Let $A \subseteq S^{\infty}$ be a Borel set such that

$$
x \in A \text { and } \sum_{i=1}^{\infty}\left(d\left(x_{i}, x_{i}^{\prime}\right)\right)^{p}<\infty \quad \text { implies } x^{\prime} \in A .
$$

Then $Y \in A$ a.s. $\sigma$ if and only if $X \in A$ a.s. $P$.

(b) Let $\left(S_{1}, d_{1}\right)$ be a separable metric space and $g_{k}$ be a sequence of measurable mappings from $S^{\infty}$ into $S_{1}$ such that

$$
d_{1}\left(g_{k}\left(x^{\prime}\right), g_{k}(x)\right) \leqslant \sum_{i=1}^{\infty} C_{k, i}\left(d\left(x_{i}, x_{i}^{\prime}\right)\right)^{p}, \quad x, x^{\prime} \in S^{\infty},
$$

where $C_{k, i}$ are positive constants bounded by 1 such that $\lim _{k} C_{k, i}=0$ for each $i$.

Let $s \in S_{1}$.

Then $g_{k}(Y) \rightarrow s$ in $\sigma$-probability if and only if $g_{k}(X) \rightarrow s$ in P-probability.

(c) Let $\left\{g_{k}\right\}$ be as in (b) satisfying (2') and let $\lambda$ be a countably additive measure on $S_{1}$. Then $g_{k}(Y) \rightarrow \lambda$ in distribution if and only if $g_{k}(X) \rightarrow \lambda$ in distribution.

The proof of this theorem is exactly the same as in the case $S=R$ except for the following lemma. The sets $B_{1}, B_{2}, \ldots, B_{m}$ satisfying conditions (i), (ii) and (iii) below for $\lambda=v_{k}$ and $\varepsilon=1 / 2^{k}$ replace the intervals $\left(a_{k, j-1}, a_{k, j}\right], 1 \leqslant j \leqslant n_{k}$, in the construction of $Z_{k}$ and $W_{k}$.

LEMMA. Let $S$ be a separable metric space and let $\lambda$ be a countably additive tight probability measure on $S$. Then for each $\varepsilon>0$, there exist finitely many disjoint Borel sets $B_{1}, \ldots, B_{m}$ such that:

(i) $\lambda\left(\cup_{i=1}^{m} B_{i}\right) \geqslant 1-\varepsilon$.

(ii) $\lambda\left(\partial B_{i}\right)=0$.

(iii) $x_{1}, x_{2} \in B_{i}$ implies $d\left(x_{1}, x_{2}\right) \leqslant \varepsilon$ for all $i$.

Proof. Let $K$ be a compact set such that $\lambda(K) \geqslant 1-\varepsilon$. Let $A_{1}, \ldots, A_{m}$ be balls of radius $\varepsilon / 4$ covering $K$. For any Borel set $B$ in $S$ let $B^{\varepsilon}=\{x \in S: d(x, B) \leqslant \varepsilon\}$. Then $\partial B^{\varepsilon} \subseteq\{x \in S: d(x, B)=\varepsilon\}$. Hence, for each $i$, choose $\varepsilon_{i}, 0<\varepsilon_{i}<\varepsilon / 4$, such that $\lambda\left(\partial A_{i}^{\varepsilon_{i}}\right)=0$. Let $B_{i}=A_{i}^{\varepsilon_{i}} \cap\left(\cup_{j=1}^{i-1} A_{j}^{\varepsilon_{j}}\right)^{c}$. Then $B_{i}$ satisfy the required conditions.

REMARK. Let $\mu_{k}$ be a sequence of tight finitely additive measures on $R$. Consider the restriction $\mu$ of the independent strategic measure with marginals $\mu_{k}$ to $\underline{B}^{\infty}$. Then $\mu$ is tight. Let $v, v_{k}$ be the countably additive measures associated with $\mu, \mu_{k}$ respectively. Then $v=\otimes_{k} v_{k}$.

Acknowledgements. The author thanks Professor A. Maitra, Professor B. V. Rao and Dr. S. Ramakrishnan for their valuable suggestions and the discussions he had with them.

\section{REFERENCES}

1. D. J. Aldous, Limit theorems for sub-sequences of arbitrarily dependent sequences of random variables, Z. Wahrsch. Verw. Gebeite 40 (1977), 59-82.

2. P. Billingsley, Convergence of probability measure, Wiley, New York, 1968.

3. L. Breiman, Probability, Addison-Wesley, California, 1968.

4. R. Chen, A finitely additive version of Kolmogorov's law of iterated logarithm, Israel J. Math. 23 (1976), 209-220. 
5. (1977), 341-356.

6. L. E. Dubins, On Lebesgue like extensions of finitely additive measures, Ann. Probab. 2 (1974), 456-463.

7. L. E. Dubins and L. J. Savage, How to gamble if you must: Inequalities for stochastic processes, McGraw-Hill, New York, 1965.

8. M. Loeve, Probability theory, 3rd ed., Van Nostrand, Princeton, N. J., 1963.

9. J. Neveu, Mathematical foundations of the calculus of probability, Holden-Day, San Francisco, Calif., 1965.

10. R. A. Purves and W. D. Sudderth, Some finitely additive probability, Ann. Probab. 4 (1976), 259-276.

11. S. Ramakrishnan, Central limit theorems in a finitely additive setting (preprint).

12. V. Strassen, An invariance principle for the law of iterated logarithm, Z. Wahrsch. Verw. Gebiete 3 (1964), 211-226.

Statistics-Mathematics Division, Indian Statistical Institute, Calcutta 700035, India

Current address: Department of Statistics, The University of North Carolina at Chapel Hill, Chapel Hill, North Carolina 27514 